\title{
24. DATA REPORT: CALCAREOUS NANNOFOSSILS OF HOLE 893A IN THE SANTA BARBARA BASIN ${ }^{1}$
}

\author{
Wuchang $\mathrm{Wei}^{2}$ and Alyssa Peleo-Alampay ${ }^{2}$
}

\section{INTRODUCTION}

Ocean Drilling Program Site $893\left(34^{\circ} 17.0^{\prime} \mathrm{N}, 120^{\circ} 02.2^{\prime} \mathrm{W}\right.$; water depth $=538 \mathrm{~m}$ ) in Santa Barbara Basin, California, is the first drillsite designed specifically for scientific studies in the California borderland. Hole 893A was cored from seafloor to $196.5 \mathrm{~m}$ below seafloor (mbsf) with a nominal core recovery rate of over $100 \%$. Hole $893 \mathrm{~B}$ was cored from the seafloor to $68.8 \mathrm{mbsf}$ with a core recovery rate similar to that in Hole 893A. This report documents calcareous nannofossils from Hole 893A.

Previously Bukry (1981) studied nannofossils from Deep Sea Drilling Project sites offshore southern California, whereas Winter (1985) studied living calcareous nannoplankton in the California Current system of southern California. However, no nannofossil studies have been published for Santa Barbara Basin. This study was carried out as a reconnaissance investigation of the nannofossils in Santa Barbara Basin. This report documents the stratigraphic distribution of nannofossils in the core-catcher samples, establishes nannofossil biostratigraphy, and briefly compares the nannofossil assemblages with those in the deep sea.

\section{METHODS}

Smear slides were made from unprocessed samples and examined with a light microscope at a magnification of $1000 \times$. Approximately 200 fields of view were searched for each slide. Abundances of nannofossils were estimated using the criteria modified from Gartner (1992):

$\mathrm{V}=$ very abundant $>25$ specimens per field of view;

$\mathrm{A}=$ abundant; $6-25$ specimens per field of view;

$\mathrm{C}=$ common; $1-5$ specimens per field of view;

$\mathrm{F}=$ few; 1 specimen per $2-10$ fields of view;

$\mathrm{R}=$ rare; 1 specimen per $11-50$ fields of view;

$\mathrm{T}=$ trace; 1 specimen per $51-200$ fields of view.

The preservation of the nannofossils was recorded as:

$\mathrm{G}=$ good; specimens exhibit little or no dissolution and/or overgrowth;

$\mathrm{M}=$ moderate; specimens exhibit moderate dissolution and/or overgrowth, and identification of species is generally not impaired.

Taxonomic references and illustrations of the species used here are in Perch-Nielsen (1985).

'Kennett, J.P., Baldauf, J.G., and Lyle, M. (Eds.), 1995. Proc. ODP. Sci. Results, 146 (Pt. 2): College Station, TX (Ocean Drilling Program).

Scripps Institution of Oceanography, University of California, San Diego, CA 92093-0215, U.S.A.

\section{RESULTS}

The stratigraphic distribution of nannofossils in Hole 893A is presented in Table 1. Emiliania huxleyi is abundant to common from Samples 146-893A-1H-CC through 9H-CC, and few to rare from Samples 146-893A-10H-CC through 21H-CC. The beginning acme of this species can thus be placed between Samples 146-893A-9H$\mathrm{CC}$ and $10 \mathrm{H}-\mathrm{CC}$. This datum has previously been calibrated with oxygen isotope Stage 4 in the deep sea (Thierstein et al., 1977), with an age of about $0.065 \mathrm{Ma}$ in the time scale of Imbrie et al. (1984). As the first occurrence of E. huxleyi is known to correlate with oxygen isotope Stage 8 (Thierstein et al., 1977), the presence of this species in all of the samples in Hole 893A suggests that the bottom of the hole is younger than oxygen isotope Stage $8(<0.28 \mathrm{Ma}$ in the time scale of Imbrie et al., 1984). These nannofossil biostratigraphic results agree with the oxygen isotope stratigraphy of Kennett (this volume), who assigned oxygen isotope Stage 4 to Cores 146-893A-9H and $10 \mathrm{H}$, and Stage 6 to the bottom of the hole.

Nannofossils are significantly less abundant in Hole 893A than in a typical deep-sea site, partly due to dilution. The assemblages are dominated by Gephyrocapsa and Emiliania huxleyi. Coccolithus pelagicus, a cool-water species, is also consistently present in all the samples examined. The most apparent difference between these assemblages and those typically found in the deep sea is that Florisphaera profunda and Umbilicosphaera sibogae are generally rare and sporadic in Hole 893A whereas they are generally abundant in the deep sea. However, no shallow-water taxa, such as Braarudosphaera, were found in the samples examined. Nannofossil species diversity is low (generally 6-7 species) in Hole 893A compared with those generally reported from low to middle latitudes. It is similar to that in the outer California continental borderland, where Bukry (1981) reported nannofossil assemblages of 2 to 10 species. Lower species diversity is also clear in the living nannoplankton study of Winter (1985) in the California Current system. Low diversities are expected because of the low temperatures of the California Current and of upwelling of cool intermediate waters. It is not clear from the present data whether species diversities or the abundances of individual species are correlated with the glacial-interglacial cycles. Quantitative and higher resolution studies should be able to address this question.

Reworked nannofossil species occur sparsely in Hole 893A. Most of these reworked specimens are of Paleogene age. The youngest reworked species recognized are Reticulofenestra pseudoumbilicus and Sphenolithus abies, both are of early Pliocene age. No reworked Cretaceous species were found.

\section{ACKNOWLEDGMENTS}

We thank J.P. Kennett for his helpful review. This study was supported in part by NSF OCE91-15786. Acknowledgment is also made to the Donors of the Petroleum Research Fund, administered by the American Chemical Society, for partial support of this research. 
Table 1. Distribution of calcareous nannofossils in Hole 893A.

\begin{tabular}{|c|c|c|c|c|c|c|c|c|c|c|c|c|c|c|c|c|c|c|c|c|c|c|c|c|c|c|c|c|c|c|c|c|}
\hline Sample & & 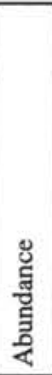 & 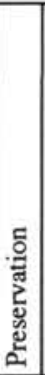 & 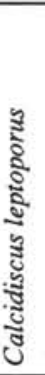 & 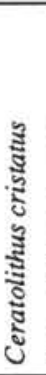 & 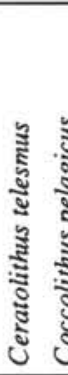 & 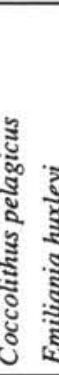 & हू & 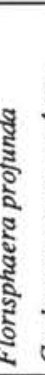 & 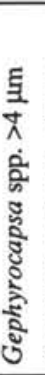 & 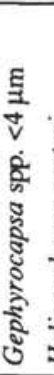 & 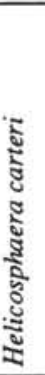 & 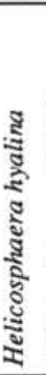 & 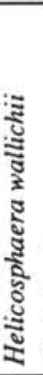 & 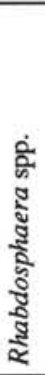 & 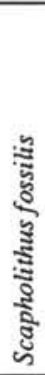 & 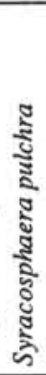 & 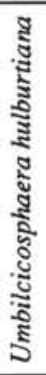 & 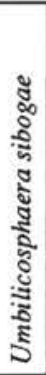 & 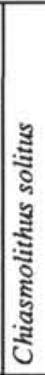 & 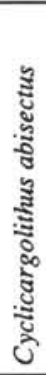 & 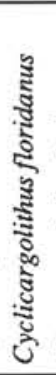 & 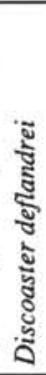 & 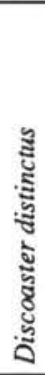 & 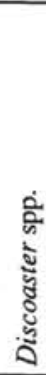 & 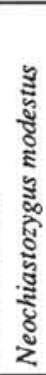 & 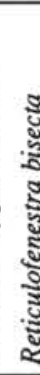 & 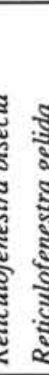 & & 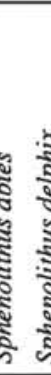 & 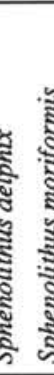 & \\
\hline & & A & G & $\mathrm{R}$ & $\mathrm{T}$ & .7 & $\mathrm{TA}$ & A 1 & $\mathrm{~F}$ & F & A & $\mathrm{R}$ & . & - & . & $T$ & $\mathrm{~T}$ & . & $\mathrm{T}$ & & . & - & & & & & & & & & & \\
\hline 2H-CC & 6.0 & V & G & . & $\mathrm{T}$ & . & $\mathrm{T} A$ & A 1 & $\mathrm{~F}$ & F & A & $\mathrm{R}$ & . & $\mathrm{T}$ & $\mathrm{T}$ & . & $\mathrm{R}$ & & . & & & . & 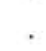 & & & & . & & & & & \\
\hline & & A & G & . & . & . & $\mathrm{F}$ & C & $\mathrm{R}$ & $\mathrm{R}$ & A & $\mathrm{R}$ & . & & . & . & . & . & . & & & . & $\mathrm{T}$ & $\mathrm{T}$ & $\mathrm{T}$ & . & & $\Gamma$ & & & & \\
\hline & & A & C & $\mathrm{T}$ & . & . & $R C$ & 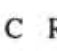 & $\mathrm{R}$ & $\mathrm{R}$ & A & . & . & $\mathrm{T}$ & . & . & . & & . & & & . & & & & & & & . & $\mathrm{T}$ & & \\
\hline & & A & $C$ & $\mathrm{~T}$ & . & . & $\mathrm{F} \quad \mathrm{C}$ & C I & $\mathrm{F}$ & $\mathrm{T}$ & A & $\mathrm{T}$ & . & $\mathrm{T}$ & . & . & . & & . & & & . & & & & & & & & & & \\
\hline & & A & C & $\mathrm{T}$ & . & . & $\mathrm{R} C$ & C F & $\mathrm{R}$ & $\mathrm{F}$ & A & . & . & 1 & $\cdot$ & . & $\mathrm{T}$ & . & . & & & . & & & & & & & & & & \\
\hline $7 \mathrm{H}-\mathrm{CC}$ & & c & 1 & & . & . & $\mathrm{R}$ & $C \mathrm{~F}$ & $\mathrm{R}$ & . & C & . & $\mathrm{T}$ & & 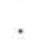 & 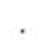 & . & & . & & & & & & & & & & & & & \\
\hline $8 \mathrm{H}-\mathrm{CC}$ & & A & C & . & . & . & $\mathrm{R}$ & $\mathrm{C} \quad \mathrm{F}$ & $\mathrm{R}$ & $\mathrm{F}$ & A & . & $\mathrm{T}$ & $\mathrm{T}$ & . & . & . & $T$ & . & & . & $\mathrm{T}$ & & & & & . & & & & & $\mathrm{T}$ \\
\hline & & A & C & 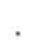 & . & . & $\mathrm{T}$ & C F & $\mathrm{R}$ & . & A & . & . & & . & . & $\mathrm{T}$ & & . & & & . & & & & & . & & & & & \\
\hline & & c & C & $\cdot$ & . & . & $\mathrm{F}$ & $\mathrm{F}$. & . & . & A & . & . & & . & . & $\mathrm{R}$ & & . & & & & & & & & & & & & & \\
\hline & & c & $c$ & $\mathrm{R}$ & . & . & $\mathrm{F}$ & $\mathrm{F}$ & . & . & A & $\mathrm{R}$ & . & 1 & . & . & $\mathrm{R}$ & $\cdot$ & . & . & 1 & $\mathrm{~T}$ & & & & & & & & & & \\
\hline $12 \mathrm{H}-\mathrm{CC}$ & & C & C & $\mathrm{T}$ & . & . & $\mathrm{R}$ & $\mathrm{F}$ & . & . & C & . & $\mathrm{T}$ & & . & . & . & . & . & & ${ }^{\circ}$ & $\mathrm{T}$ & & & & & & & & & & \\
\hline $13 \mathrm{H}-\mathrm{CC}$ & 20.5 & $\mathrm{~F}$ & $N$ & 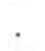 & . & . & $\mathrm{T}$ & $\mathrm{R}$ & . & $\mathrm{T}$ & $\mathrm{R}$ & 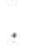 & 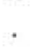 & & . & . & . & 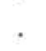 & . & & & . & & & & & & & & $\mathrm{T}$ & & \\
\hline & & C & M & $\mathrm{T}$ & . & $\mathrm{T}$ & $\mathrm{F}$ & $\mathrm{R} \quad \mathrm{F}$ & $\mathrm{R}$ & $\mathrm{T}$ & $\mathrm{C}$ & . & . & & . & . & . & . & $\mathrm{T}$ & & 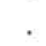 & $\mathrm{T}$ & & & & & & & & & & \\
\hline & & $\mathrm{F}$ & N & $\mathrm{T}$ & . & . & $\mathrm{T} F$ & $\mathrm{R}$ & . & $\mathrm{T}$ & $\mathrm{R}$ & . & . & & . & . & . & . & . & & . & $\mathrm{R}$ & & & & & & & & & & \\
\hline & & A & M & $\mathrm{F}$ & . & . & $\mathrm{R}$ & $\mathrm{F} \quad \mathrm{F}$ & $\mathrm{F}$ & $\mathrm{F}$ & $\mathrm{C}$ & . & $\mathrm{T}$ & $\mathrm{T}$ & . & . & . & . & . & & 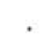 & . & 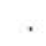 & & & 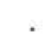 & . & & . & & & \\
\hline & & A & N & $\mathrm{R}$ & . & . & $\mathrm{F}$ & $\mathrm{R} F$ & $\mathrm{R}$ & $\mathrm{R}$ & A & . & $\mathrm{T}$ & & . & . & . & . & . & & . & . & & & & . & & & $\mathrm{T}$ & & & \\
\hline $18 \mathrm{H}-\mathrm{CC}$ & & A & $\mathrm{N}$ & $\mathrm{T}$ & . & . & F & R $\mathrm{F}$ & $\mathrm{R}$ & . & A & . & $\mathrm{T}$ & & . & $\mathrm{T}$ & $\mathrm{T}$ & - & . & & - & $\mathrm{T}$ & & & & & & & & $\mathrm{R}$ & & 1 \\
\hline $19 \mathrm{H}$ & & C & M & . & . & . & $\mathrm{F}$ & $\mathrm{R}$. & . & . & $\mathrm{C}$ & 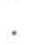 & . & & . & . & . & . & . & & 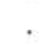 & . & & & & 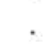 & & & $R$ & & & \\
\hline & & $\mathrm{F}$ & M & $\mathrm{R}$ & . & . & F F & $\mathrm{R}$ & . & $\mathrm{R}$ & $\mathrm{C}$ & . & $\mathrm{T}$ & & . & . & . & . & . & & & $\mathrm{T}$ & & & & . & & & . & & & . \\
\hline 1H-CC & 96.5 & $\mathrm{~F}$ & $\mathrm{M}$ & F & . & . $\mathrm{F}$ & $\mathrm{R} F$ & $\mathrm{R}$ & . & $\mathrm{R}$ & $\mathrm{C}$ & & & & . & . & $\mathrm{T}$ & & $\mathrm{T}$ & & $\mathrm{T}$ & $\mathrm{T}$ & & & & 1 & & & & & & \\
\hline
\end{tabular}

Note: Reworked species are tabulated on right.

\section{REFERENCES}

Bukry, D., 1981. Pacific coast coccolith stratigraphy between Point Conception and Cabo Corrientes, Deep Sea Drilling Project Leg 63. In Yeats, R.S., Haq. B.U., et al., Init. Repts. DSDP, 63: Washington (U.S. Govt. Printing Office), 445-471.

Gartner, S., 1992. Miocene nannofossil chronology in the North Atlantic, DSDP Site 608. Mar. Micropaleontol., 18:307-331.

Imbrie, J., Hays, J.D., Martinson, D.G., McIntyre, A., Mix, A.C., Morley, J.J., Pisias, N.G., Prell, W.L., and Shackleton, N.J., 1984. The orbital theory of Pleistocene climate: support from a revised chronology of the marine $\delta^{18} \mathrm{O}$ record. In Berger, A., Imbrie, J., Hays, J., Kukla, G., and Saltzman, B. (Eds.), Milankovitch and Climate (Pt. 1): Dordrecht (D. Reidel), 269-305.
Perch-Nielsen, K., 1985. Cenozoic calcareous nannofossils. In Bolli, H.M. Saunders, J.B., and Perch-Nielsen, K. (Eds.), Plankton Stratigraphy: Cambridge (Cambridge Univ. Press), 427-554.

Thierstein. H.R., Geitzenauer, K., Molfino, B., and Shackleton, N.J., 1977. Global synchroneity of late Quaternary coccolith datum levels: validation by oxygen isotopes. Geology. 5:400-404.

Winter, A., 1985. Distribution of living coccolithophores in the California Current system, southern California Borderland. Mar. Micropaleontol., 9:385-393.

Date of initial receipt: 19 January 1994

Date of acceptance: 4 November 1994

Ms 146SR-305 